\title{
Modified Algorithm for Solving Linear Integro-Differential Equations of the Second Kind
}

\author{
M. Al-Towaiq, Ahmed Kasasbeh \\ Department of Mathematics and Statistics, Jordan University of Science and Technology, Al Ramtha, Irbid, Jordan \\ Email: towaiq@just.edu.jo,just1966@yahoo.com
}

How to cite this paper: Al-Towaiq, M. and Kasasbeh, A. (2017) Modified Algorithm for Solving Linear Integro-Differential Equations of the Second Kind. American Journal of Computational Mathematics, 7, 157-165. https://doi.org/10.4236/ajcm.2017.72014

Received: May 4, 2017

Accepted: June 19, 2017

Published: June 22, 2017

Copyright (c) 2017 by authors and Scientific Research Publishing Inc. This work is licensed under the Creative Commons Attribution International License (CC BY 4.0).

http://creativecommons.org/licenses/by/4.0/

c) (i) Open Access

\begin{abstract}
In this paper, a modified algorithm is proposed for solving linear integro-differential equations of the second kind. The main idea is based on applying Romberg extrapolation algorithm (REA), on Trapezoidal rule. In accordance with the computational perspective, the comparison has shown that Adomian decomposition approach is more effective to be utilized. The numerical results show that the modified algorithm has been successfully applied to the linear integro-differential equations and the comparisons with some existing methods appeared in the literature reveal that the modified algorithm is more accurate and convenient.
\end{abstract}

\section{Keywords}

Algorithm, Integro-Differential Equations, Trapezoidal Rule, Romberg Extrapolation

\section{Introduction}

Mathematical modelling of real-life problems usually results in functional equations, such as differential, integral, and integro-differential equations. Many mathematical formulations of physical phenomena reduced to integro-differential equations, like fluid dynamics, biological models and chemical kinetics [1] [2] [3].

The numerical solution of integro-differential equation is a part of numerical analysis, which has been changed by the ongoing revolution in numerical methods. With the development of technology, useful methods are evolving for full utilization of the inherent powers of high speed and large memory computing machine. Many significant methods were discovered to approximate the solution 
of linear integro-differential equations, such as the Improved Bessel collocation method [4], the Legendre wavelets method [5], the finite element method [6], the Tau method [7], Euler polynomials [8], Sherman-Morrison formula [9], the Adomian's decomposition method [10] [11], the Cas wavelet [12], the homotopy perturbation method [13], the Variational iteration method [14] [15] [16], the comined Laplace transform and the Adomian decomposition methods [17], the Sinc method [18] [19], the Galerkin method [20], Romberg extrapolation [21], the Chebyshev polynomial approach [22] [23], Lagrange Interpolation [24], and many mathematicians still search to get strong methods and powerful techniques to solve problems in integro-differential equations.

Without loss of generality, the linear Fredholmintegro differential equation of the second kind was considered.

$$
u^{\prime}(x)=f(x)+\int_{a}^{b} k(x, t) u(t) \mathrm{d} t, u(a)=\alpha,
$$

where, $\alpha$ is a real constant.

Some authors studied the numerical solution of the nonlinear integro-differential equations by the Adomian decomposition method and compared it with the variational iteration method [14] [15] [16]. Therefore, results show that the variation iteration method (VIM) has been successfully employed to obtain the approximate analytical solutions of the nonlinear integro-differential equations. Others solve it through a comparison of the Adomian decomposition method and the wavelet-Galerkin method [20]. From the computational view point, the comparison shows that the Adomian decomposition method is more efficient and easy to use.

Saadati et al. [1], presented numerical method to approximate the integrodifferential equations (Volterra, Fredholm) using the Trapezoidal rule. This method based on transforming the first derivative integro-differential equations to a system of algebraic equations.

In [22] [23], the Chebyshev polynomial was used to approximate the solution of integral equations, system of higher-order linear Fredholm-Volterra integro-differential equations, and integro-differential equations. The main idea of their techniques based on transforming these equations to a system of algebraic equations. They showed that the method has some major advantages: Chebyshev coefficients of the solution are found very easily and this process is very fast. An interesting feature of the method obtained analytical solution in many cases. Many studies have indicated the variational iteration method to solve the linear and nonlinear integro differential equations (Volterra, Fredholm) [3] [14] [15] [16] [17]. They applied the variational iteration method to approximate the solutions of the integro-differential equations. The results show that this method is very effective with low computation time. Also, some authors concluded that the method can be used to find exact solution for some cases.

M. T. Rashed [24] computed the solution of the integro-differential equation numerically by using the Lagrange interpolation. It is being concluded that the method is the very rapid convergence, and successfully treated Volterra type and 
Fredholm type without any difficulties. In this paper, the solutions of the linear integro-differential equations of different types are studied, analyzed and implemented using a modified algorithm based on the Romberg extrapolation techniques.

Jaradat et al. [25] presented an applicability of the Homotopy method to solve Fredholm integro-differential equation. They test the validity and the applicability of this method, and show that the Homotopy techniques are very powerful to approximate the linear Fredholm integro-differential equations.

Mostafa Nadir and Azedine Rahmoune [26], presented a numerical method to approximate the solution of the linear Volterra integral equations of the second kind, based on Simpson's rule.

The paper is organized as follows: In Section 2, the proposed technique for solving the linear integro-differential equations is introduced. Some numerical experiments are presented in Section 3. The paper is concluded in Section 4.

\section{The Modified Algorithm}

In this section, the chosen algorithm is introduced for the solution of Fredholm integro-differential equation of the second kind. First, the Trapezoidal rule is applied to approximate the integral and the finite difference to approximate the derivative in (1), and then Romberg extrapolation is applied to increase the accuracy of the solution.

The numerical setting and the approximation of the integral for the Volterra Equation (1) will result in the coefficient matrix of the linear system of equations being a lower triangular one, which is exactly due to the variable upper limit $\mathrm{x}$ of the integration in (1), because in this equation the kernel $k(x, t) \equiv 0$ for $t>x$ as the integrand can be considered identically zero above it is upper limit of integration $x$. So, for the discrete case $k\left(x_{i}, t_{j}\right)=k_{i j}=0$ for $j>i$ is used. Then the system of linear equations with such a natural triangular coefficient matrix can be solved easily.

To use Trapezoidal rule the interval of integration $(a, x)$ has been partitioned into $\mathrm{n}$ equally spaced subintervals of width

$$
h=\frac{x_{n}-a}{n}, n \geq 1,
$$

where $x_{n}$ is the end point chosen for $x$, set $t_{0}=$ a should be set and

$$
t_{j}=a+j h=t_{0}+j h, j=1,2,3, \cdots, n .
$$

Then the approximation of the integral in the integro-differential Equation (1) is given by,

$$
\int_{a}^{x} k(x, t) u(t) \mathrm{d} t \cong h\left[\frac{1}{2} k\left(x, t_{0}\right) u\left(t_{0}\right)+k\left(x, t_{1}\right) u\left(t_{1}\right)+\cdots+k\left(x, t_{n-1}\right) u\left(t_{n-1}\right)+\frac{1}{2} k\left(x, t_{n}\right) u\left(t_{n}\right)\right],
$$

where,

$$
h=\frac{t_{j}-a}{j}=\frac{x-a}{n}, t_{j} \leq x, j \geq 1, x=x_{n}=t_{n} .
$$


Replace $n$ by $2^{n}=m$, then (4) becomes,

$$
\int_{a}^{x} k(x, t) u(t) \mathrm{d} t \cong h\left[\frac{1}{2} k\left(x, t_{0}\right) u\left(t_{0}\right)+k\left(x, t_{1}\right) u\left(t_{1}\right)+\cdots+k\left(x, t_{m-1}\right) u\left(t_{m-1}\right)+\frac{1}{2} k\left(x, t_{m}\right) u t_{m}\right],
$$

which is called $R(n, 0)$. Then Romberge algorithm (REA) $R_{k, j}$ can be applied, where,

$$
R_{k, j}=R_{k, j-1}+\frac{R_{k, j-1}-R_{k-1, j-1}}{4^{j}-1}, i, j \geq 1
$$

Now, substitute Equation (6) in (1), thus obtain,

$$
u^{\prime}(x)=f(x)+h\left[\frac{1}{2} k\left(x, t_{0}\right) u\left(t_{0}\right)+k\left(x, t_{1}\right) u\left(t_{1}\right)+\cdots+k\left(x, t_{m-1}\right) u\left(t_{m-1}\right)+\frac{1}{2} k\left(x, t_{m}\right) u\left(t_{m}\right)\right],
$$

If $n$ values of $u_{i}^{\prime}=u^{\prime}\left(x_{i}\right)=u^{\prime}\left(t_{i}\right)$ are considered and,

$$
k\left(x, t_{j}\right) u\left(t_{j}\right)=k\left(x_{i}, t_{j}\right) u\left(t_{j}\right), i=1,2,3, \cdots, n,
$$

then Equation (8) becomes,

$$
u^{\prime}\left(x_{i}\right)=f\left(x_{i}\right)+h\left[\frac{k\left(x_{i}, t_{0}\right) u\left(t_{0}\right)}{2}+\frac{k\left(x_{i}, t_{m}\right) u\left(t_{m}\right)}{2}+k\left(x_{i}, t_{1}\right) u\left(t_{1}\right)+\cdots+k\left(x_{i}, t_{m-1}\right) u\left(t_{m-1}\right)\right]
$$

For simplicity Equation (9) becomes,

$$
u_{i}^{\prime}=f_{i}+h\left[\frac{k_{i 0} u_{0}}{2}+\frac{k_{i, m} u_{m}}{2}+k_{i 1} u_{1}+\cdots+k_{i, m-1} u_{m-1}\right] .
$$

The finite difference formula is applied,

$$
u^{\prime}(x) \cong \frac{3 u(x)-4 u(x-h)+u(x-2 h)}{2 h}
$$

to approximate $u_{i}^{\prime}(x)$ in Equation (10) to get the following equation

$$
\frac{3 u_{m}-4 u_{m-1}+u_{m-2}}{2 h}=f_{i}+h\left[\frac{k_{i 0} u_{0}}{2}+\frac{k_{i, m} u_{m}}{2}+k_{i 1 u 1}+\cdots+k_{i, m-1} u_{m-1}\right] \text {. }
$$

In matrix notation, Equation (11) transform into the following system of linear equations:

$$
K U=F,
$$

where,

$$
\begin{aligned}
& K=\left[(-) h^{2} k_{11}\left(1-2 h^{2} k_{12}\right)\left(-2 h^{2} k_{13}\right) \cdots-h^{2} k_{1_{m}}\right. \\
& -\left(2 h^{2} k_{21}+1\right)-2 h^{2} k_{22}\left(1-2 h^{2} k_{23}\right)\left(1-2 h^{2} k_{23}\right)\left(1-2 h^{2} k_{23}\right) \cdots-h^{2} k_{12 m} \\
& -2 h^{2} k_{31}-\left(2 h^{2} k_{32}+1\right)-h^{2} k_{33} \\
& \vdots \\
& -2 h^{2} k_{m-1,1}-2 h^{2} k_{m-1,2} \cdots-2 h^{2} k_{m-1, m-1}\left(1-2 h^{2} k_{m-1, m}\right) \\
& \left.-2 h^{2} k_{m, 1}-2 h^{2} k_{m, 2} \cdots-\left(2 h^{2} k_{m, m-1}+4\right)-\left(2 h^{2} k_{m, m}-3\right)\right] \\
& U=\left(u_{1}, u_{2}, \cdots, u_{m-1}, u_{m}\right)^{t}
\end{aligned}
$$


And,

$$
F=\left[2 h f_{1}+\left(h^{2} k_{10}+1\right) u_{0}, 2 h f_{2}+\left(h^{2} k_{20}\right) u_{0}, \cdots, 2 h f_{m-1}+h^{2} k_{m-1,0}\left(u_{0}\right), 2 h f_{m}+h^{2} k_{m-1,0}\left(u_{0}\right)\right]^{t} \text {. }
$$

This system can be solved for the unknowns $u_{i}^{\prime}$ s, $i=1,2, \cdots, m$ easily.

\section{Numerical Experiments}

In this paper, illustrative examples of integro-differential equation are given to demonstrate the accuracy and efficiency of the proposed technique and compare it with some other existing methods.

Example 3.1 [10]. Consider the following Linear Fredholm integro-differential equation of the first derivative:

$$
u^{\prime}(x)=1-\frac{1}{3} x+\int_{0}^{1} x t u(t), u(0)=0
$$

In Equation (16),

$$
f(x)=1-\frac{1}{3} x, k(x, t)=x t .
$$

The exact solution of Equation (3.1) is:

$$
u(x)=x .
$$

The modified algorithm of Romberg extrapolation is applied for solving this example. Following equation is used:

$$
\begin{gathered}
h=h_{5}=\frac{1}{16} \text {, then } k=5 \text {, with } x_{0}=0 \text {, and } x_{16}=1 \text {, with mesh points, } \\
x_{i}=i h_{5}, i=1,2, \cdots, 16 .
\end{gathered}
$$

Apply Equation (10) and Equation (11) to compute the approximate solution,

$$
u_{i}, i=1,2, \cdots, 16 \text {. }
$$

Table 1 shows the absolute errors of the numerical results of $R_{5,2}$. It is shown that the proposed algorithm is accurate and efficient. Based on the recursive relations of Romberg extrapolation the accuracy increased with less computation time.

In [10], Vahidi reported the computed absolute error for Example (3.1), with $n=10$ for different methods such as the CAS wavelet method, the differential transform method (DTM), and the Adomian decomposition method (ESA). For comparison purposes, norm 2 for the absolute error vector of all the points on [0, $1]$ is computed. The computed norms for all the above mentioned methods and technique (REA) are shown in Table 2. The table shows that algorithm is more accurate and convenient than other methods. But, if technique is compared with the VIM method, the VIM gives better accuracy.

However, the technique has the advantage, on the time $R_{k, 1}$ is computed, the accuracy will increase with less computation as the recursive relation of Romberg increases.

Example 3.2 [1]. In this example, the technique for solving the following Linear Volterra integro-differential equation of the first derivative is applied: 
Table 1. Approximate solution and absolute errors of Example (3.1), using $R_{5,2}$.

\begin{tabular}{cccc}
\hline$x_{i}$ & $u_{i}$ using Romberg $R_{5,2}$ & Exact value of $u\left(x_{i}\right)$ & Absolute error \\
\hline 0.0625 & 0.0625015 & 0.0625 & $1.45 \times 10^{-6}$ \\
0.125 & 0.125 & 0.125 & 0 \\
0.1875 & 0.187513 & 0.1875 & $1.3 \times 10^{-5}$ \\
0.25 & 0.250005 & 0.25 & $5 \times 10^{-6}$ \\
0.3125 & 0.312536 & 0.3125 & $3.6 \times 10^{-5}$ \\
0.375 & 0.375662 & 0.375 & $6.6 \times 10^{-4}$ \\
0.4375 & 0.437571 & 0.4375 & $7.1 \times 10^{-5}$ \\
0.5 & 0.500022 & 0.5 & $2.1 \times 10^{-5}$ \\
0.5625 & 0.562618 & 0.5625 & $1.1 \times 10^{-4}$ \\
0.625 & 0.625684 & 0.625 & $6.8 \times 10^{-4}$ \\
0.6875 & 0.687676 & 0.6875 & $1.7 \times 10^{-4}$ \\
0.75 & 0.750084 & 0.75 & $4.8 \times 10^{-5}$ \\
0.8125 & 0.812746 & 0.8125 & $2.4 \times 10^{-4}$ \\
0.875 & 0.875717 & 0.875 & $7.1 \times 10^{-4}$ \\
0.9375 & 0.937827 & 0.9375 & $3.2 \times 10^{-4}$ \\
1 & 1.00008 & 1 & $8.3 \times 10^{-5}$ \\
\hline
\end{tabular}

Table 2. Absolute error.

\begin{tabular}{cc}
\hline The method & Norm of the absolute errors \\
\hline REA & $1.2 \times 10^{-3}$ \\
CAS Wavelet & $3.7 \times 10^{-2}$ \\
DTM & $1.7 \times 10^{-1}$ \\
ESA & $1.1 \times 10^{-3}$ \\
\hline
\end{tabular}

$$
u^{\prime}(x)=1+\sin x+\int_{0}^{x} u(t), u(0)=-1,
$$

In Equation (19), $f(x)=1+\sin x, k(x, t)=1$. The exact solution of Equation (3.3) is

$$
u(x)=\frac{1}{4} \mathrm{e}^{x}-\frac{3}{4} \mathrm{e}^{-x}-\frac{1}{2} \cos x
$$

The modified algorithm of Romberg extrapolation is applied for solving this example.

The study use $h_{5}=\frac{1}{16}$, then, $k=5$, with $x_{0}=0$, and $x_{16}=1$, with mesh points,

$$
x_{i}=i h_{5}, i=1,2, \cdots, 16 .
$$

Apply Equation (11) to compute the approximate solution $u_{i}, i=1,2, \cdots, 16$.

Table 3 shows the absolute errors of the numerical results of $R_{5,2}$. It is shown that the proposed algorithm is accurate and efficient. Based on the recursive relations of Romberg extrapolation the accuracy increased with less computation 
time.

In [10], Vahidi reported the computed absolute error for Example 3.2, with $n=$ 10 for the different methods mentioned in example 3.1. The computed norms for all the methods are shown in Table 4. The table shows that the technique is more accurate than the CAS wavelet and the differential transform method (DTM) and has the same accuracy as the Adomian decomposition method (ESA).

\section{Conclusion}

A modified technique by using Romberg extrapolation on the Trapezoidal rule was introduced to find an approximate solution of the linear integro-differential equation. Some numerical examples appearing in the literature are presented for introducing the main idea behind the approach and for comparisons purposes. The numerical results show that the technique has been successfully applied to the linear integro-differential equations with first derivative. Comparisons with the methods provided in [10] revealed that the technique is more accurate and

Table 3. Approximate solution and absolute errors of Example (3.2) using $R_{5,2}$.

\begin{tabular}{|c|c|c|c|}
\hline$x_{i}$ & $u_{\mathrm{i}}$ using Romberg $R_{5,2}$ & Exact value of $u\left(X_{i}\right)$ & Absolute error \\
\hline 0.0625 & -0.939313 & -0.93746 & $1.85306 \times 10^{-3}$ \\
\hline 0.125 & -0.877167 & -0.874684 & $2.48294 \times 10^{-3}$ \\
\hline 0.1875 & -0.816836 & -0.811451 & $5.38507 \times 10^{-3}$ \\
\hline 0.25 & -0.752159 & -0.74755 & $4.60856 \times 10^{-3}$ \\
\hline 0.3125 & -0.691295 & -0.682786 & $8.50879 \times 10^{-3}$ \\
\hline 0.375 & -0.623507 & -0.616973 & $6.53375 \times 10^{-3}$ \\
\hline 0.4375 & -0.561194 & -0.549936 & $1.12583 \times 10^{-2}$ \\
\hline 0.5 & -0.489657 & -0.481509 & $8.14771 \times 10^{-3}$ \\
\hline 0.5625 & -0.425194 & -0.411536 & $1.36583 \times 10^{-2}$ \\
\hline 0.625 & -0.349485 & -0.339866 & $9.59152 \times 10^{-3}$ \\
\hline 0.6875 & -0.282077 & -0.266357 & $1.57202 \times 10^{-2}$ \\
\hline 0.75 & -0.201611 & -0.190869 & $1.07417 \times 10^{-2}$ \\
\hline 0.8125 & -0.130719 & -0.11327 & $1.74494 \times 10^{-2}$ \\
\hline 0.875 & -0.0451471 & -0.0334261 & $1.17209 \times 10^{-2}$ \\
\hline 0.9375 & -0.0299532 & -0.0487906 & $1.88374 \times 10^{-2}$ \\
\hline 1 & -0.121113 & -0.13351 & $1.23963 \times 10^{-2}$ \\
\hline
\end{tabular}

Table 4. Absolute error.

\begin{tabular}{cc}
\hline The method & Norm of the absolute errors \\
\hline REA & $8.1 \times 10^{-3}$ \\
CAS Wavelet & $7.8 \times 10^{-2}$ \\
DTM & $1.5 \times 10^{-1}$ \\
ESA & $3.7 \times 10^{-3}$ \\
\hline
\end{tabular}


convenient than the other methods. When the technique is compared with the VIM, the VIM normally gives a better accuracy than the method selected. However, the technique has one advantage: the accuracy can be increased with less computation as the recursive relations of Romberg increases.

\section{Acknowledgements}

The authors are very thankful to all the associated personnel in any reference that contributed in/for the purpose of this research. Further, this research holds no conflict of interest and is not funded through any source.

\section{References}

[1] Saadati, R., Raftari, B., Abibi, H., Vaezpour, S.M. and Shakeri, S. (2008) A Comparison between the Variational Iteration Method and Trapezoidal Rule for Solving Linear Integro-Differential Equations. World Applied Sciences Journal, 4, 321-325.

[2] Jerri, A.J. (1999) Introduction to Integral Equations with Applications. John Wiley \& Sons, New York.

[3] Alawneh, A., Al-Khaled, K. and Al-Towaiq, M. (2010) Reliable Algorithms for Solving Integro-Differential Equations with Applications. International Journal of Computer Mathematics, 87, 1538-1554. https://doi.org/10.1080/00207160802385818

[4] Yuzbas, S. (2016) Improved Bessel Collocation Method for Linear VolterraintegroDifferential Equations with Piecewise Intervals and Application of a Volterra Population Model. Applied Mathematical Modelling, 40, 5349-5363. https://doi.org/10.1016/j.apm.2015.12.029

[5] Razzaghi, M. and Yousefi, S. (2005) Legendre Wavelets Method for the Nonlinear Volterra-Fredholm Integral Equations. Mathematics and Computers in Simulation, 70, 1-8. https://doi.org/10.1016/j.matcom.2005.02.035

[6] Sharma, N. and Sharma, K. (2015) Finite Element Method for a Nonlinear Parabolicintegro-Differential Equation in Higher Spatial Dimensions. Applied Mathematical Modelling, 39, 7338-7350. https://doi.org/10.1016/j.apm.2015.02.037

[7] Pour-Mahmoud, J., Rahimi-Ardabili, M.Y. and Shahmorad, S. (2005) Numerical Solution of the System of Fredholm Integro-Differential Equations by the Tau Method. Applied Mathematics and Computation, 168, 465-478. https://doi.org/10.1016/j.amc.2004.09.026

[8] Mirzaee, F. and Bimesl, S. (2015) Numerical Solutions of Systems of High-Order Fredholmintegro-Differential Equations Using Euler Polynomials. Applied Mathematical Modelling, 39, 6767-6779. https://doi.org/10.1016/j.apm.2015.02.022

[9] Egidi, N. and Maponi, P. (2010) The Use of Sherman-Morrison Formula in the Solution of Fredholmintegral Equation of Second Kind. Mathematics and Computers in Simulation, 81, 693-704. https://doi.org/10.1016/j.matcom.2010.03.006

[10] Vahidi, A.R., Babolian, E., Cordshooli, G.A. and Azimzadeh, Z. (2009) Numerical Solution of Fredholm Integro-Differential Equation by Adomian's Decomposition Method. International Journal of Mathematical Analysis, 36, 1769-1773.

[11] El-Sayed, S.M. and Abdel-Aziz, M.R. (2003) A Comparison of Adomian's Decomposition Method and Wavelet-Galerkin Method for Solving Integro-Differential Equations. Applied Mathematics and Computation, 136, 151-159. https://doi.org/10.1016/S0096-3003(02)00024-3

[12] Danfu, H. and Xufeng, S. (2007) Numerical Solution of Integro-Differential Equa- 
tions by Using CAS Wavelet Operational Matrix of Integration. Applied Mathematics and Computation, 194, 460-466. https://doi.org/10.1016/j.amc.2007.04.048

[13] Yusufoglu, E. (2009) Improved Homotopy Perturbation Method for Solving Fredholm Type Integro-Differential Equations. Chaos Solitons Fractals, 41, 28-37. https://doi.org/10.1016/j.chaos.2007.11.005

[14] Wang, S.Q. and He, J.H. (2007) Variational Iteration Method for Solving IntegroDifferential Equations. Physics Letters A, 367, 188-191. https://doi.org/10.1016/j.physleta.2007.02.049

[15] Abbasbandy, S. and Shivanian, E. (2009) Application of Variational Iteration Method for $n$ th-Order Integro-Differential Equations. Zeitschrift für Naturforschung $A, 64,439-444$. https://doi.org/10.1515/zna-2009-7-805

[16] Sweilam, N.H. (2007) Fourth Order Integro-Differential Equations Using Variational Iteration Method. Computers \& Mathematics with Applications, 54, 1086 1091. https://doi.org/10.1016/j.camwa.2006.12.055

[17] Wazwaz, A.M. (2010) The Combined Laplace Transform-Adomian Decomposition Method for Handling Nonlinear Volterra Integro-Differential Equations. Applied Mathematics and Computation, 216, 1304-1309. https://doi.org/10.1016/j.amc.2010.02.023

[18] Rashidinia, J. and Zarebnia, M. (2007) The Numerical Solution of Integro-Differential Equation by Means of the Sinc Method. Applied Mathematics and Computation, 188, 1124-1130. https://doi.org/10.1016/j.amc.2006.10.063

[19] Kajani, M.T., Ghasemi, M. and Babolian, E. (2006) Numerical Solution of Linear Integro-Differential Equation by Using Sine-Cosine Wavelets. Applied Mathematics and Computation, 180, 569-574. https://doi.org/10.1016/j.amc.2005.12.044

[20] Maleknejad, K. and Kajani, M.T. (2004) Solving Linear Integro-Differential Equation System by Galerkin Methods with Hybrid Functions. Applied Mathematics and Computation, 159, 603-612. https://doi.org/10.1016/j.amc.2003.10.046

[21] Meštrović, M. and Ocvirk, E. (2007) An Application of Romberg Extrapolation on Quadrature Method for Solving Linear Volterra Integral Equations of the Second Kind. Applied Mathematics and Computation, 194, 389-393. https://doi.org/10.1016/j.amc.2007.04.043

[22] Daşcioğlu, A. (2006) A Chebyshev Polynomial Approach for Linear FredholmVolterra Integro-Differential Equations in the Most General Form. Applied Mathematics and Computation, 181, 103-112. https://doi.org/10.1016/j.amc.2006.01.018

[23] Daşcioğlu, A. and Sezer, M. (2005) Chebyshev Polynomial Solutions of Systems of Higher-Order Linear Fredholm-Volterra Integro-Differential Equations. Journal of the Franklin Institute, 342, 688-701. https://doi.org/10.1016/j.jfranklin.2005.04.001

[24] Rashed, M.T. (2004) Lagrange Interpolation to Compute the Numerical Solutions of Differential, Integral and Integro-Differential Equations. Applied Mathematics and Computation, 151, 869-878. https://doi.org/10.1016/S0096-3003(03)00543-5

[25] Jaradat, H., Alsayyed, O. and Al-Shara, S. (2008) Numerical Solution of Linear Integro-Differential Equations. Journal of Mathematics and Statistics, 4, 250-254. https://doi.org/10.3844/jmssp.2008.250.254

[26] Mostafa, N. and Azedine, R. (2007) Modified Method for Solving Linear Volterra Integral Equations of the Second Kind Using Simpson's Rule. International Journal: Mathematical Manuscripts, 1, 141-146. 
Submit or recommend next manuscript to SCIRP and we will provide best service for you:

Accepting pre-submission inquiries through Email, Facebook, LinkedIn, Twitter, etc. A wide selection of journals (inclusive of 9 subjects, more than 200 journals)

Providing 24-hour high-quality service

User-friendly online submission system

Fair and swift peer-review system

Efficient typesetting and proofreading procedure

Display of the result of downloads and visits, as well as the number of cited articles Maximum dissemination of your research work

Submit your manuscript at: http://papersubmission.scirp.org/

Or contact ajcm@scirp.org 